\title{
Avances en el desarrollo de una vacuna efectiva contra Cryptosporidium parvum: una revisión de la literatura
}

\author{
David R Salamanca1; Jessica S Molina-Franky; Anny J Camargo-Mancipe; \\ Alida M Gómez-Rodríguez ${ }^{1}$
}

\section{RESUMEN}

Introducción. Cryptosporidium parvum es un parásito zoonótico altamente prevalente, asociado a enfermedad diarreica en población inmunocomprometida, niños y terneros menores de 30 días. Esta infección puede ocasionar deshidratación, alteración del estado de conciencia, retraso en el desarrollo global y, en algunos casos, la muerte del paciente. A pesar de la alta prevalencia de C. parvum, no existen medicamentos completamente efectivos ni una vacuna aprobada para prevenir dicha enfermedad.

Objetivo. Realizar una revisión de la literatura sobre candidatos vacunales contra C. parvum.

Método. Revisión documental mediante la búsqueda de la literatura de los últimos 20 años, disponible en las bases de datos PubMed central, WEB OF SCIENCE, Embase, REDALYC y LILACS.

Resultados. Las vacunas atenuadas, recombinantes, basadas en ADN, expresadas en vectores bacterianos y sintéticas han mostrado resultados prometedores en la inducción de inmunogenicidad contra los antígenos de C. parvum, siendo el antígeno de superficie de 15 kilodaltons de Cryptosporidium parvum (cp15), el antígeno inductor de una mejor respuesta inmune celular y humoral en el modelo murino estudiado.

Conclusión. Se espera que la incorporación de nuevas técnicas para la selección de antígenos promisorios y la ejecución de una gran cantidad de ensayos in vivo, favorezcan el desarrollo de una vacuna totalmente efectiva contra C. parvum. Aunque el camino para lograr este objetivo será largo y difícil, se convierte en la mejor alternativa para controlar una de las enfermedades de interés en salud pública, con mayor impacto en la población inmunocomprometida.

Palabras clave: Cryptosporidium parvum, vacunas sintéticas, vacunas de ADN, inmunogenicidad vacunal, antígeno CP15.

${ }^{1}$ Universidad de Boyacá, Tunja, Boyacá.

Correspondencia: David Ricardo Salamanca. Dirección Carrera $2^{a}$ este Nº 64-169. Tunja.

Celular: 3132423136.

Correo electrónico: drsalamanca@uniboyaca.edu.co

Citar este artículo así:

Salamanca DR, Molina-Franky JS, Camargo-MancipeAJ, Gómez-RodríguezAM. Avances en el desarrollo de una vacuna efectiva contra Cryptospo-ridium parvum: una revisión de la literatura. Revista Investig Salud Univ Boyacá. 2020;7(1): 118-136. doi: https:// doi.org/10.24267/23897325.373 


\title{
Advances in the development of an effective vaccine against Cryptosporidium parvum: An review of the literature
}

\begin{abstract}
Introduction. Cryptosporidium parvum is a highly prevalent zoonotic parasite, associated with diarrheal disease in immunocompromised population, children and calves under 30 days. This infection is associated to dehydration, delayed global development and, in some cases, the death of the patient. Despite the high prevalence of $C$. parvum, there are no fully effective medications and an approved vaccine to prevent such disease.

Objective. To conduct a thorough review of the literature on vaccine candidates against C. parvum. Method Documentary review by searching the literature of the last 20 years, available in the central PubMed, WEB OF SCIENCE, Embase, REDALYC and LILACS databases.

Results. Attenuated, recombinant, DNA-based, expressed in bacterial vectors and synthetic vaccines have shown promising results in inducing immunogenicity against $C$. parvum, being the Cryptosporidium parvum 15 kiloDalton surface antigen (cp15), the antigen inducer of a better cellular and humoral immune response in the murine model studied.

Conclusion. It is expected that the incorporation of new techniques for the selection of promising antigens and the execution of a large number of in vivo assays will favor the development of a fully effective vaccine against $C$. parvum. Although the way to achieve this goal will be long and difficult, it will become the best alternative to control one of the diseases with the greatest impact on the immunocompromised population.
\end{abstract}

Key words: Cryptosporidium parvum, vaccines, synthetic, vaccines, DNA, vaccines, subunit, immunogenicity, vaccine, CP15 protein. 


\title{
Avanços no desenvolvimento de uma vacina eficaz contra Cryptosporidium parvum: uma revisão da literatura
}

\begin{abstract}
RESUMO
Introdução. O Cryptosporidium parvum é um parasita zoonótico de alta prevalência associado à doença diarreica em populações imunocomprometidas, crianças e bezerros com menos de 30 dias. Essa infecção pode causar desidratação, alteração do estado de consciência, atraso no desenvolvimento global e, em alguns casos, a morte do paciente. Apesar da alta prevalência de C. parvum, não existem medicamentos totalmente eficazes e uma vacina aprovada para prevenir a doença.

Objetivo. Realizar uma revisão literária dos candidatos à vacina contra C. parvum.

Método. Revisão documental, mediante pesquisa da literatura dos últimos 20 anos, disponível nas bases de dados PubMed central, WEB OF SCIENCE, Embase, REDALYC e LILACS.

Resultados. Vacinas atenuadas, recombinantes e baseadas em DNA, expressas em vetores bacterianos e sintéticos, mostraram resultados promissores na indução de imunogenicidade contra antígenos de C. parvum, sendo o antígeno de superfície de 15 kilodaltons de Cryptosporidium parvum (cp15) o antígeno indutor de uma melhor resposta imune celular e humoral no modelo murino estudado.

Conclusão. Se espera que a incorporação de novas técnicas para a seleção de antígenos promissores e a execução de um grande número de ensaios in vivo favoreçam o desenvolvimento de uma vacina totalmente eficaz contra C. parvum. Embora o caminho para alcançar este objetivo seja longo e difícil, torna-se a melhor alternativa para controlar uma das doenças de interesse na saúde pública com maior impacto na população imunocomprometida.

Palavras-chave: Cryptosporidium parvum, vacinas sintéticas, vacinas de DNA, imunogenicidade da
\end{abstract} vacina, antígeno CP15. 


\section{INTRODUCCIÓN}

Según la Organización Mundial de la Salud (OMS), la enfermedad diarreica representa aproximadamente el $10,5 \%$ de los 8 millones de muertes que se registran anualmente, a nivel mundial, en niños menores de 5 años. En orden de frecuencia, la enfermedad diarreica es ocasionada principalmente por rotavirus, Cryptosporidium spp., Shigella y Escherichia coli enterotoxigénica (1). De los anteriores, Cryptosporidium spp. es el único agente etiológico para el cual no se dispone de vacuna, ni de un tratamiento totalmente efectivo $(1,2)$. Crypstosporidium parvum es un protozoo intracelular zoonótico perteneciente al phylum Apicomplexa que afecta diversas especies animales, especialmente bovinos menores de 30 días $(3,4)$, población humana inmunocomprometida, como pacientes con SIDA o cáncer y también población infantil menor de 5 años $(5,6)$.

Los ooquistes de Cryptosporidium spp. son pequeños, miden entre 4 a $6 \mu \mathrm{m}$ de diámetro y tienen una forma esférica-ovoide. El ciclo de vida de Cryptosporidium se completa dentro de un solo hospedero, presentando dos fases: sexual y asexual, para lo cual se llevan a cabo las siguientes seis etapas de desarrollo: 1) desenquistamiento (liberación de los esporozoítos), 2) merogonia, 3) gametogonia, 4) fertilización y desarrollo del cigoto, 5) la formación de paredes de ooquistes ambientalmente resistentes y 6 ) la esporogonia $(7,8)$.
Existe una amplia gama de mecanismos de transmisión para este patógeno, ya sea por la ingesta de ooquistes a través del contacto con objetos previamente expuestos a materia fecal, o por el consumo de alimentos y agua contaminada $(9,10)$. Cada ooquiste contiene cuatro esporozoítos, estos ooquistes son ambientalmente estables, capaces de sobrevivir al tratamiento rutinario de aguas residuales y resistentes a la inactivación con desinfectantes comúnmente utilizados para potabilizar el agua (2).

Una vez ingeridos los ooquistes, en el intestino delgado (íleon) se produce el desenquistamiento que da lugar a la liberación cuatro esporozoítos, los cuales buscarán invadir a los enterocitos. Los esporozoítos se fijan a la superficie luminal de los enterocitos y se diferencian asexualmente en trofozoítos que producen dos tipos diferentes de merontes por merogonia (11). Los merontes tipo I producen ocho merozoítos que invaden las células epiteliales vecinas y se convierten en merontes tipo II o completan otro ciclo de merontes tipo I. Los merontes tipo II producen cuatro merozoítos dando lugar a los gamontes (microgametos o macrogametos) (12). La fertilización entre gamontes produce la formación de un cigoto que se convierte en un ooquiste que es excretado en las heces(13) (Imagen 1). 
Imagen 1. Ciclo de vida de C. parvum. (1) El ooquiste es ingerido por el hospedero a partir de agua o alimentos contaminados. Al ingresar al tracto gastrointestinal, cada ooquiste libera 4 esporozoítos que infectan las células epiteliales y maduran dando lugar a las formas parasitarias de trofozoito, merontes tipo I y II, microgamentos, macrogametos y finalmente, un nuevo ooquiste (2), el cual al ser eliminado por las heces del hospedero (3) puede infectar nuevas fuentes hídricas y alimentos, propagando así la infección de C. parvum.

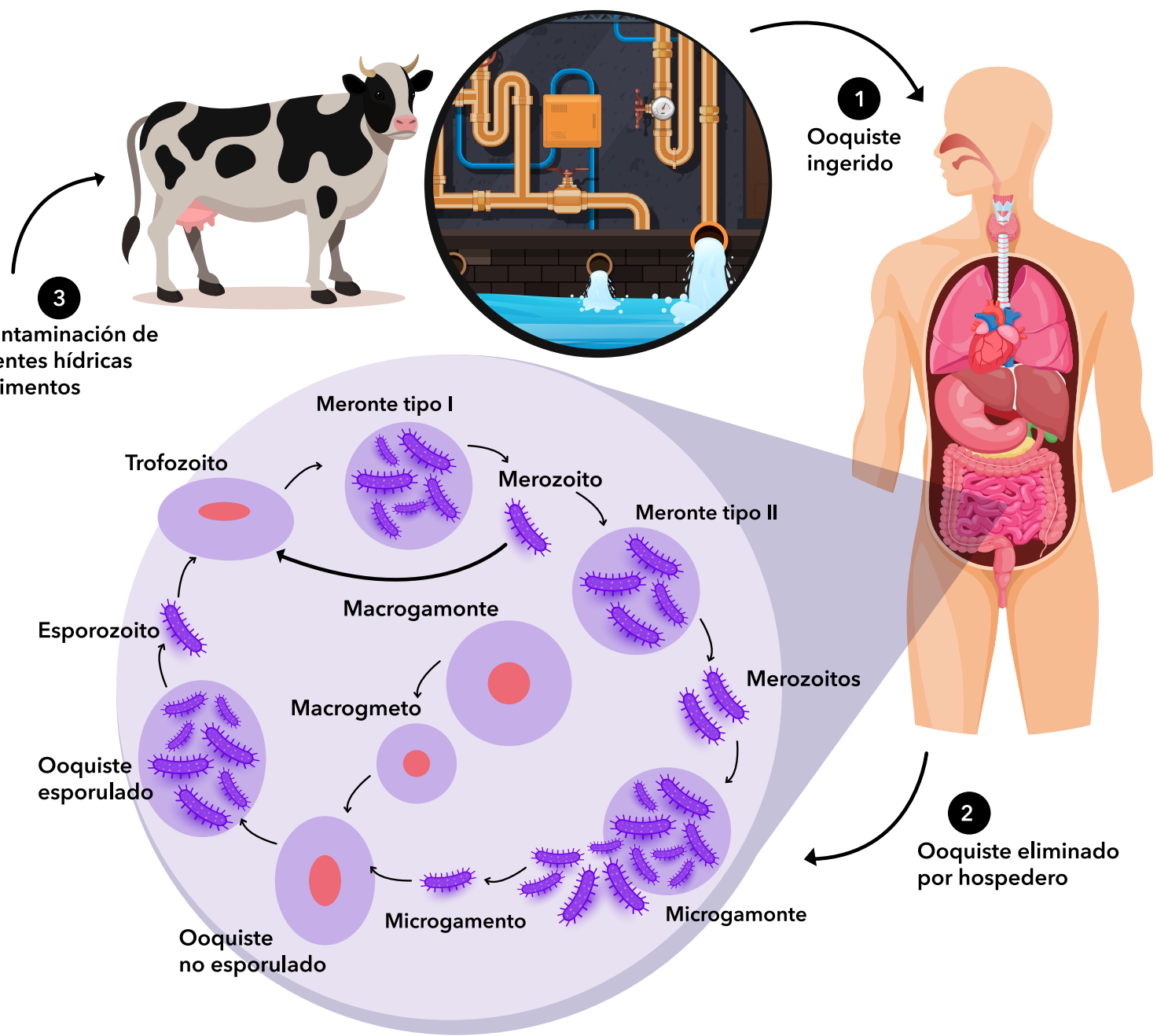

Fuente: Autor 
En el campo veterinario, la criptosporidiosis induce diarrea acuosa profusa y deshidratación, asociada con altas tasas de morbimortalidad, incremento en el costo de atención médica veterinaria e importantes pérdidas económicas (14-16), con lo cual se afecta en gran medida el desarrollo del sector agropecuario.

En los pacientes inmunocomprometidos se ha reportado diarrea persistente y una mortalidad 2 a 3 veces mayor en comparación a la población general (17). Debido a la capacidad para contaminar ambientes por la eliminación de ooquistes a través de las heces y al acúmulo de los mismos en las fuentes hídricas; esto sumado a la resistencia frente a la desinfección estándar con cloro, Cryptosporidium parvum es considerado un problema de salud pública (18).

La prevalencia de Cryptosporidium parvum entre los niños con diarrea, en países en vías de desarrollo como Brasil, Colombia, Venezuela, Indonesia, Tailandia, Sudáfrica, Ghana y la India, varía entre el $3 \%$ y el $37 \%$; por el contrario, en las partes desarrolladas del mundo como Gran Bretaña, Estados Unidos, Canadá, Australia y Dinamarca, representa solo el 1-4\% de la diarrea infantil (19).

Entre las diversas infecciones oportunistas, Cryptosporidium parvum es uno de los agentes comunes para los individuos infectados por el VIH. La criptosporidiosis sintomática es más común en aque-
Ilos pacientes con recuento linfocitos T CD $4<200$ células / mm3 (20-22). Su prevalencia en estos pacientes en América del Norte varía entre el $3,5 \%$ y $11,9 \%$, mientras que en América del Sur es del $4 \%$ al $22,8 \%$ (19).

A pesar de los recientes avances para el manejo de infecciones de interés médico, no existe una terapia efectiva para el control de Crypstosporidium spp. en población vulnerable (23), por lo cual, esta infección puede ocasionar complicaciones médicas dadas por la deshidratación, alteración del estado de conciencia, retraso en el desarrollo global $y$, en algunos casos, la muerte del paciente $(24,25)$. Teniendo en cuenta la problemática que representa la propagación de $C$. parvum para la salud humana y animal, diferentes candidatos a vacuna han sido estudiados con el fin de encontrar una solución profiláctica contra esta enfermedad. En términos generales, los candidatos a vacuna contra C. parvum reportados a la fecha se dividen en cinco categorías: 1. vacunas atenuadas; 2 . vacunas recombinantes; 3 . vacunas basadas en ADN; 4. antígenos expresados en vectores bacterianos, y 5 . vacunas sintéticas. En el presente manuscrito se describen los estudios más representativos de los candidatos a vacuna que han tenido resultados prometedores y de los cuales derivan nuevos ensayos experimentales. 


\section{METODOLOGÍA}

Se realizó una revisión exhaustiva de la literatura sobre los candidatos a vacuna contra $C$. parvum. La búsqueda se hizo a partir de fuentes secundarias de información, es decir, artículos y libros relacionados con esta patología, publicados en las bases de datos electrónicas como PubMed central, WEB OF SCIENCE, Embase, REDALYC y LILACS. Con base en los Medical Subject Headings (MeSH), se seleccionaron las palabras clave para la búsqueda en idiomas español e inglés, en combinación con Cryptosporidium parvum, Vaccines, Synthetic, Vaccines, DNA, Vaccines, Subunit, Vaccine, CP15 protein.

Fueron seleccionados todos los artículos en el idioma inglés y español, publicados entre 1999 y 2019, se escogieron 82 artículos por título y resumen, de estos se excluyeron 31 que presentaban artículo completo, pero no incluían en forma detallada los resultados de inmnunogenicidad y/o protección final. En total, se analizaron 51 artículos que presentaban la información metodológica detallada para el desarrollo de los candidatos a vacuna descritos.

\section{RESULTADOS}

Con base a la búsqueda realizada, se presenta a continuación un análisis detallado del ciclo de vida de C. parvum y los grupos de candidatos a vacuna más representativos contra esta enfermedad (Tabla 1).

\section{Candidatos a vacunas contra C. parvum Vacunas atenuadas}

Debido a la dificultad para propagar continuamente $C$. parvum in vitro, los intentos de atenuar el parásito han sido limitados. Un método que se ha utilizado es el tratamiento con irradiación gamma de ooquistes o esporozoítos $(26,27)$.

Para evaluar la respuesta de inmunidad protectora contra la criptosporidiosis, ooquistes de la cepa de Cryptosporidium parvum lowa se expusieron a varios niveles de irradiación gamma (350-500 Gy) y se inocularon en terneros de un día de edad, en los cuales se examinaron diariamente los signos clínicos y paraclínicos de la enfermedad. Las dosis más altas de irradiación (450 o 500 Gy) impidieron el desarrollo de ooquistes, pero las terneras permanecieron susceptibles a la infección por $C$. parvum. Los ooquistes de Cryptosporidium parvum expuestos a $400 \mathrm{~Gy}$ fueron incapaces de cualquier desarrollo medible, pero conservaron la capacidad de provocar una respuesta protectora contra la exposición a este parásito. Estos hallazgos indican que puede ser posible proteger a los terneros contra la criptosporidiosis mediante inoculación con ooquistes de $C$. parvum expuestos a una irradiación gamma de 400 Gy (28). 
Tabla 1. Resumen de resultados de los principales candidatos a vacuna contra C. parvum.

\begin{tabular}{|c|c|c|c|c|c|c|}
\hline $\begin{array}{l}\text { Candidato a } \\
\text { vacuna }\end{array}$ & $\begin{array}{l}\text { Tipo de } \\
\text { vacuna }\end{array}$ & Antígeno & $\begin{array}{l}\text { Vía de adminis- } \\
\text { tración /Dosis }\end{array}$ & Participantes & Principales resultados & Ref. \\
\hline $\begin{array}{l}\text { Ooquistes de C. } \\
\text { parvum Gam- } \\
\text { ma-Irradiated }\end{array}$ & $\begin{array}{l}\text { Vacuna } \\
\text { atenuada }\end{array}$ & $\begin{array}{l}\text { Ooquistes de C. } \\
\text { parvum irradiados }\end{array}$ & Oral & $\begin{array}{c}8 \text { terneros } \\
\text { menores de } 30 \\
\text { días de edad }\end{array}$ & $\begin{array}{l}\text { La administración de ooquistes de } \\
\text { C. parvum expuestos a irradiación } \\
\text { gamma }>450 \text { Gy previene la inva- } \\
\text { sión del parásito. }\end{array}$ & 28 \\
\hline Cp12 / Cp21 & $\begin{array}{c}\text { Vacuna } \\
\text { basada en } \\
\text { ADN }\end{array}$ & $\begin{array}{l}\text { Antígeno de es- } \\
\text { porozoito de C. } \\
\text { parvum de } 12 \text { y } 21 \\
\mathrm{kDa}(\mathrm{cp} 11 \text { y } \mathrm{Cp} 21)\end{array}$ & $\begin{array}{c}\text {-Intra-muscular } \\
\text {-Intra-nasal } \\
(100 \mu \mathrm{g} / \\
3 \text { dosis })\end{array}$ & $\begin{array}{c}200 \text { ratones } \\
\text { BALB / c de 5-7 } \\
\text { semanas de edad }\end{array}$ & $\begin{array}{l}\text { La inmunización intra-nasal de las } \\
\text { proteínas Cp12-Cp21 en ratones } \\
\text { redujo en } 77.5 \% \text { la generación de } \\
\text { nuevos ooquistes de C. parvum. }\end{array}$ & 31 \\
\hline CP15-ADN & $\begin{array}{c}\text { Vacuna } \\
\text { basada en } \\
\text { ADN }\end{array}$ & $\begin{array}{l}\text { Antígeno de es- } \\
\text { porozoito de C. } \\
\text { parvum de } 15 \mathrm{kDa}\end{array}$ & $\begin{array}{l}\text { Intra-nasal } \\
\text { (50 } \mu \mathrm{g} / \\
3 \text { dosis })\end{array}$ & $\begin{array}{l}5 \text { ratones BALB } \\
\text { / c hembra de } 8 \\
\text { semanas de edad }\end{array}$ & $\begin{array}{l}\text { El ADN plasmídico puede pro- } \\
\text { porcionar alta respuesta humoral } \\
\text { (títulos de anticuerpos IgG: 1600) } \\
\text { y celular a las infecciones por C. } \\
\text { parvum en mamíferos. }\end{array}$ & 33 \\
\hline $\mathrm{Cp} 23$ & $\begin{array}{c}\text { Vacuna } \\
\text { basada en } \\
\text { ADN }\end{array}$ & $\begin{array}{l}\text { Antígeno de es- } \\
\text { porozoito de C. } \\
\text { parvum de } 23 \mathrm{kDa}\end{array}$ & $\begin{array}{l}\text { Subcutánea } \\
\qquad(100 \mu \mathrm{g} / \\
3 \text { dosis })\end{array}$ & $\begin{array}{c}20 \text { ratones } \\
\text { hembra de 6-8 } \\
\text { semanas de edad }\end{array}$ & $\begin{array}{l}\text { La vacunación con ADN de Cp23 } \\
\text { indujo una reducción del 50-60\% } \\
\text { en el desprendimiento de oocis- } \\
\text { tos, lo que indica una protección } \\
\text { parcial contra la infección por C. } \\
\text { parvum. }\end{array}$ & 35 \\
\hline $\begin{array}{l}\text { Salmonella } \\
\text { typhimurium } \\
\text { atenuada que } \\
\text { codifica los } \\
\text { antígenos Cp23 } \\
\text { y Cp40 }\end{array}$ & $\begin{array}{l}\text { Vacuna que } \\
\text { usa vectores } \\
\text { de bacterias } \\
\text { atenuadas }\end{array}$ & $\begin{array}{l}\text { Antígeno de es- } \\
\text { porozoito de C. } \\
\text { parvum de } 23 \mathrm{kDa} \\
\text { (cp23) y } 40 \mathrm{KDa} \\
\text { (Cp40) }\end{array}$ & 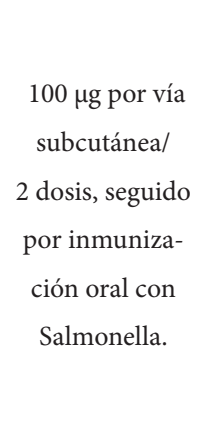 & $\begin{array}{c}16 \text { ratones } \\
\text { C57BL / } 6 \text { de 6-8 } \\
\text { semanas de edad }\end{array}$ & $\begin{array}{l}\text { La inoculación oral con S. Typhi- } \\
\text { murium SL3261 recombinante } \\
\text { puede inducir alta respuesta de } \\
\text { anticuerpos específicos al antígeno } \\
\text { Cp23 o Cp40 de C. parvum en } \\
\text { ratones. } \\
\text { 30\% de anticuerpos IgA contra } \\
\text { Cp23 detectados en suero y } \\
\text { mucosa (heces). }\end{array}$ & 40 \\
\hline
\end{tabular}




\begin{tabular}{|c|c|c|c|c|c|c|}
\hline $\begin{array}{l}\text { Candidato a } \\
\text { vacuna }\end{array}$ & $\begin{array}{l}\text { Tipo de } \\
\text { vacuna }\end{array}$ & Antígeno & $\begin{array}{l}\text { Vía de adminis- } \\
\text { tración /Dosis }\end{array}$ & Participantes & Principales resultados & Ref. \\
\hline $\begin{array}{l}\text { C. parvum p23 } \\
\text { recombinante }\end{array}$ & $\begin{array}{l}\text { Vacuna re- } \\
\text { combinante }\end{array}$ & $\begin{array}{l}\text { Antígeno de es- } \\
\text { porozoito de C. } \\
\text { parvum de } 23 \mathrm{kDa} \\
\text { (cp23) expresado } \\
\text { en E. coli }\end{array}$ & 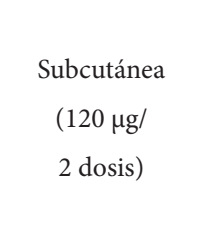 & 20 cabras & $\begin{array}{l}\text { P23 es un antígeno importante que } \\
\text { estimula las respuestas inmunita- } \\
\text { rias del huésped, principalmente } \\
\text { en la producción de anticuerpos } \\
\text { específicos bajo infección natural. }\end{array}$ & 48 \\
\hline
\end{tabular}

Fuente: Autor

\section{Vacunas basadas en ADN}

Los métodos basados en ADN implican la administración de un plásmido que codifica un antígeno particular. El plásmido es absorbido por las células huésped y el antígeno se expresa y se expone al sistema inmune. Varios estudios recientes han evaluado las vacunas basadas en ADN para las proteínas de Cryptosporidium parvum 2 (P2), C. parvum 12 (Cp12), C. parvum 15/60 (Cp15 / 60), C. parvum 23 (Cp23) у C. parvum 21 (Cp21), sin embargo, CP15 ha sido la más estudiada por diferentes autores (29-32).

La primera vacuna de ADN reportada codifica un antígeno de esporozoíto de C. parvum de $15 \mathrm{kDa}$ (CP15-ADN), clonado en el plásmido pcDNA3se. A partir de esta vacuna, un grupo de ratones $B A L B / C$ de ocho semanas de edad fue inmunizado por vía intranasal, con lo cual se produjo una respuesta específica y duradera de IgA anti-CP15 en las secreciones intestinales y de IgG específica en suero, 3 meses y 1 año después de la primera inoculación de ADN. Los ratones inmunizados con ADN de CP15 también desarrollaron una respuesta proliferativa de linfocitos $T$ específicos de antígeno, tanto en el bazo como en los ganglios linfáticos mesentéricos. Estos resultados indican que el ADN plasmídico puede proporcionar un medio poderoso para provocar respuestas humorales y celulares intestinales a las infecciones por C. parvum en mamíferos (33).

En otro ensayo, para estudiar las respuestas inmunitarias humorales y celulares inducidas por la proteína de esporozoíto de C. parvum CP15 / 60 , se inyectó por vía intramuscular el plásmido recombinante que contiene el gen CP15 / 60 en ratones $B A L B / c$. Las respuestas inmunes humorales y celulares se detectaron en diferentes momentos después de la inmunización. Los resultados experimentales han demostrado que el plásmido recombinante puede inducir respuestas inmunitarias específicas $y$, por lo tanto, proteger a los ratones del reto con los ooquistes, lo que sugiere que el plásmido recombinante podría ser 
un candidato potencial de la vacuna de ADN (34). Por otra parte, la administración de una vacuna de ADN que codifica C. parvum Cp15 y Cp23 dio como resultado la inducción de respuestas inmunitarias Th1, así como una mayor resistencia a la infección (29). La eficacia de las vacunas de ADN también se ha demostrado mediante la generación de respuestas inmunitarias específicas de Cp23: los ratones inmunizados con ADN de Cp23 desarrollaron protección parcial contra la infección por $C$. parvum, como lo demuestra la reducción $>60 \%$ en la eliminación de ooquistes después del reto (35).

Más recientemente, un estudio evaluó a la proteína ácida ribosomal P2 ( $C p \mathrm{P} 2)$ de $C$. parvum como potencial candidato a vacuna, a partir de la inmunización subcutánea en el oído de ratones con el ADN que codifica para esta proteína, clonado en el vector pUMVC4b. Se concluyó que el antígeno CpP2 es capaz de proporcionar un medio eficaz para provocar respuestas humorales y celulares y tiene el potencial de generar inmunidad protectora contra la infección por C. parvum, pero puede requerir el uso de vectores o adyuvantes alternativos para producir una respuesta más potente y equilibrada (36-38).

\section{Uso de vectores de bacterias atenuadas}

Los vectores bacterianos son muy prometedores para la administración del antígeno de una vacuna, ya que pueden provocar respuestas inmunitarias protectoras contra patógenos bacterianos, virales y protozoarios en ratones y humanos (39).

La cepa SL3261 de la vacuna de Salmonella enterica serovar Typhimurium atenuada se utilizó, por primera vez, como un sistema de administración de antígenos para la inmunización oral de ratones contra dos antígenos de C. parvum, Cp23 y Cp40, subclonados en el sistema vectorial pTECH1. Los ratones se inocularon por vía oral con una dosis única de SL3261 / pTECH-Cp23 o Cp40, respectivamente; de esta forma, se demostró la estabilidad del plásmido tanto in vitro como in vivo. Se detectaron anticuerpos específicos de inmunoglobulina $\mathrm{G}(\mathrm{lg} \mathrm{G})$ en suero contra el antígeno Cp23 o Cp40 35 días después de la inmunización, además, se detectaron anticuerpos IgA en suero y IgA en la mucosa (heces) en el $30 \%$ de los ratones inmunizados con $\mathrm{Cp} 23$. Se demostró que una sola inoculación oral con S. typhimurium SL3261 recombinante puede inducir respuestas de anticuerpos específicos contra el antígeno Cp23 o Cp40 de C. parvum en ratones, lo que sugiere que la Salmonella recombinante es un sistema de administración factible para una vacuna contra la infección por C. parvum (40).

En otro estudio, se administraron 2 antígenos en ratones, Cp15 y profilina de Cryptosporidium, en un régimen heterólogo de refuerzo primario 
como fusiones con citolisina A (ClyA) en un vector de vacuna viva de Salmonella; a partir de allí, se encontró que estos antígenos fueron potentes en la inducción de respuestas inmunes humorales y celulares (41).

Otro sistema de vectores utilizado para la entrega del antígeno Cryptosporidium spp. es Toxoplasma gondii (42). La inmunización de ratones con $T$. gondii que expresa el antígeno C. parvum $\mathrm{P} 23$ dio como resultado altos niveles de IgG en suero, predominantemente $\lg \mathrm{G} 1$, que es característica de una respuesta inmune $\mathrm{TH} 2(43,44)$. En otro estudio, Lactobacillus casei se utilizó para administrar C. parvum P23 a ratones y generó niveles aumentados de interferón gamma (IFN $\square$ ), inter leucina 6 (IL-6), inmunoglobulina $\mathrm{G}(\mathrm{IgG})$ en suero e inmunoglobulina $A(\operatorname{IgA})$ fecal $(45,46)$.

\section{Vacunas recombinantes}

En un estudio reciente se realizó la clonación y expresión del gen gp40 / 15 de C. parvum para proporcionar proteínas recombinantes expresadas en Escherichia coli. La secuencia del gen gp40 / 15 se extrajo del GenBank y se clonó en el Plásmido PET28a + . Como resultado, la reacción en cadena de polimerasa (PCR) de colonia y los métodos de digestión enzimática mostraron un fragmento de 921 pb. Se realizaron titulaciones de los anticuerpos en suero, obtenidos de ratones inmunizados con esta proteína recombinante, los cuales fueron significativamente más altos $(P<0,0001)$ que los del grupo control, además, la titulación de anticuerpos en el grupo de estudio con cuatro inyecciones fue significativamente mayor que la de las tres inyecciones $(P<0.05)$. Por lo anterior, se considera el potencial uso de esta proteína en el desarrollo de vacunas recombinantes y kits de diagnóstico contra C. parvum (47).

En otro ensayo, se aislaron ooquistes de Cryptosporidium de terneros infectados naturalmente; para este caso, los ooquistes se purificaron y caracterizaron como C. parvum por PCR anidada; adicionalmente, para obtener la proteína P23 recombinante, se aisló el ARNm de las muestras obtenidas y sintetizó el ADNc. Como resultado, la secuenciación del producto de PCR presentó $100 \%$ de identidad con las secuencias P23 conocidas en GenBank; por otra parte, el ADNc de doble cadena P23 se clonó luego en el vector de expresión pGEX-5X-2 y se preparó la proteína recombinante P23. El análisis de transferencia mostró que P23 podría ser reconocido por el suero positivo de $C$. parvum; además, el suero de cabra inmunizada con la proteína P23 recombinante también reconoció una banda de proteína con aproximadamente $23 \mathrm{kDa}$ en lisados preparados a partir de los ooquistes.

Dado que P23 es una glucoproteína de superficie inmunodominante expresada en la fase temprana de la infección, y los epítopos inmunogénicos 
se encuentran en su cadena residual de secuencia de aminoácidos, la proteína recombinante P23 podría recomendarse como un candidato favorable para la vacunación contra la infección por C. parvum (48).

Por otra parte, los resultados de un análisis genómico comparativo indicaron que los genes que codifican dos proteasas similares a la insulina de C. parvum (INS19 e INS20), cgd6_5510 y cgd6_5520, se pierden en muchas especies de Cryptosporidium. Estos genes fueron expresados de forma recombinante en Escherichia coli para la preparación de antisuero. Una proteína de $\sim 180$ kDa de INS20-19 fue específicamente reconocida por el antisuero policlonal anti-INS19 en lisado de esporozoítos; asimismo, se observó que INS20-19 es probablemente una proteína con alta expresión en la región apical de los esporozoítos y la neutralización de la proteína condujo a una reducción parcial de la carga de parásitos en cultivos de células HCT-8 y MDBK a las $24 \mathrm{~h}$. Tomados en conjunto, estos hallazgos respaldan la participación de INS20-19 en la invasión o el proceso de desarrollo temprano de C. parvum (49).

\section{Vacunas sintéticas}

Para evaluar la respuesta inmune humoral inducida contra péptidos derivados de Cryptosporidium parvum CP15 (antígeno de superficie de esporozoito de $15 \mathrm{kDa}$ ) y proteínas CSL (antígeno de tipo circumsporozoito), un estudio tuvo como objetivo realizar pruebas preliminares en ratones albinos suizos (ICR) inmunizados con péptidos de estas proteínas. Los péptidos se identificaron y caracterizaron utilizando herramientas bioinformáticas y se sintetizaron químicamente, además, se determinó la respuesta de anticuerpos y se midió el efecto neutralizante de los anticuerpos en cultivo celular; sin embargo, a pesar de que todos los péptidos estudiados fueron capaces de estimular la producción de anticuerpos, se detectaron anticuerpos neutralizantes derivados de CP15. Por lo tanto, se recomiendan estudios adicionales destinados a evaluar aún más el potencial de los péptidos como candidatos a vacunas (50).

\section{CONCLUSIONES}

Dado que las opciones de tratamiento farmacológico son limitadas para la criptosporidiosis, y pueden no ser tan efectivas para individuos inmunodeficientes, la investigación en vacunas continúa siendo el pilar científico para prevenir esta devastadora enfermedad.

Comprender en detalle las interacciones huésped-parásito y los elementos esenciales de la inmunidad de Cryptosporidium spp. puede conducir al desarrollo de inmunoterapias o vacunas efectivas. El aumento continuo en los datos de la secuencia del genoma de $C$. parvum podría facilitar la identificación y caracterización de posibles antígenos vacunales. 
Una vacuna ideal contra Crypstosporidium debería proporcionar una respuesta inmunitaria rápida y duradera en todas las personas vacunadas, ser ampliamente protectora contra las especies y subtipos más comunes del organismo, prevenir la transmisión de enfermedades y ser fácilmente accesible, estable y económica.

Sin embargo, antes de que se pueda lograr el desarrollo de una vacuna hay que superar diferentes obstáculos científicos, logísticos y económicos asociados. Se requiere la selección de epítopes altamente inmunogénicos y de regiones conservadas del parásito que estén implicadas en los procesos de invasión a enterocitos. Así mismo, se requiere un mayor número de estudios in vitro e in vivo con distintos modelos animales (bovinos y ratones) que permitan obtener resultados fehacientes de seguridad y eficacia para el inicio de ensayos clínicos en humanos.

Por otra parte, sería interesante el análisis de una vacuna multiepítope, pues, aunque la mayoría de los estudios de vacunas se han centrado únicamente en antígenos del estadio esporozoíto de $C$. parvum, es probable que existan otros candidatos prometedores expresados en el estadio merozoíto que, al trabajarse en conjunto, sean capaces de bloquear el ciclo de vida del parásito en cualquiera de sus etapas.
Para identificar nuevos objetivos potenciales de vacuna, los investigadores deberían centrarse también en las formas de detectar proteínas necesarias para la infección y que puedan inducir respuestas inmunitarias protectoras mediadas por células. Es importante tener en cuenta que muchas de las proteínas implicadas en la unión e invasión de $C$. parvum sufren una modificación postraduccional extensa, particularmente la glicosilación. Estas modificaciones se pueden requerir para la función y el potencial inmunogénico de la proteína y, por lo tanto, es importante tenerlas en cuenta al diseñar un cribado de antígenos de Cryptosporidium.

Además, la selección de antígenos, en función de su capacidad para provocar una óptima respuesta inmune celular, debería ser un factor clave en la identificación de candidatos a vacunas. También, es probable que se necesite una vacuna que incorpore múltiples antígenos para asegurar una protección óptima.

Muchos avances nuevos en las técnicas de vacunación, como el uso de vacunas de ADN, vacunas sintéticas (basadas en mínimas subunidades) nuevos vectores que estimulan la inmunidad de la mucosa y el uso de oligonucleótidos como adyuvantes, también pueden facilitar el desarrollo de vacunas contra esta enfermedad. 
Se espera que la incorporación de nuevas técnicas para la selección de antígenos promisorios y la ejecución de una gran cantidad de ensayos in vivo, favorezcan el desarrollo de una vacuna totalmente efectiva contra $C$. parvum. Aunque el camino para lograr este objetivo será largo y difícil, se convierte en la mejor alternativa para controlar una de las enfermedades de interés en salud pública con mayor impacto en la población inmunocomprometida.

\section{LIMITACIONES}

No hubo limitaciones.

\section{CONFLICTOS DE INTERÉS}

Los autores declaran no tener conflicto de interés.

\section{FINANCIACIÓN}

Los autores declaran financiación de la Universidad de Boyacá de los medios necesarios para llevar a cabo este artículo de revisión.

\section{REFERENCIAS}

1. Striepen B. Parasitic infections: Time to tackle cryptosporidiosis. Nature. 2013;503(7475):189-91. https://doi. org/10.1038/503189a
2. Korpe PS, Valencia C, Haque $R$, Mahfuz $M$, McGrath M, Houpt E, et al. Epidemiology and Risk Factors for Cryptosporidiosis in Children From 8 Low-income Sites: Results From the MAL-ED Study. Clinical Infectious Diseases. 2018;67:16609. https://doi.org/10.1093/cid/ciy355

3. Tomazic $M L$, Maidana J, Dominguez $M$, Uriarte EL, Galarza R, Garro C, et al. Molecular characterization of Cryptosporidium isolates from calves in Argentina. Vet Parasitol. 2013;198(3-4):382-6. https://doi. org/10.1016/j.vetpar.2013.09.022

4. Chalmers RM, Davies AP, Tyler K. Cryptosporidium. Microbiology. 2019;165(5):500-2. https://doi.org/10.1099/ mic. 0.000764

5. Abubakar I, Aliyu SH, Arumugam C, Usman NK, Hunter PR. Treatment of cryptosporidiosis in immunocompromised individuals: systematic review and meta-analysis. Br J Clin Pharmacol. 2007;63(4):387-93. https://doi.org/10.1111/ j.1365-2125.2007.02873.x

6. Hossain MJ, Saha D, Antonio M, Nasrin D, Blackwelder WC, Ikumapayi UN, et al. Cryptosporidium infection in rural gambian children: Epidemiology and risk factors. PLoS Negl Trop Dis. 2019;13(7):e0007607. https:// dx.doi.org/10.1371/journal.pntd.0007607 
7. Leitch GJ, He Q. Cryptosporidiosis-an overview. J Biomed Res. 2012;25(1):1-16. https://doi. org/10.1016/S1674-8301(11)60001-8

8. Dumaine JE, Tandel J, Striepen B. Cryptosporidium parvum. Trends in Parasitology. 2019. https://doi.org/10.1016/j. pt.2019.11.003

9. Baldursson $S$, Karanis P. Waterborne transmission of protozoan parasites: review of worldwide outbreaks - an update 2004-2010. Water Res. 2011;45(20):6603-14. https://doi. org/10.1016/j.watres.2011.10.013

10. Gallas-Lindemann C, Sotiriadou I, Plutzer J, Noack MJ, Mahmoudi MR, Karanis P. Giardia and Cryptosporidium spp. dissemination during wastewater treatment and comparative detection via immunofluorescence assay (IFA), nested polymerase chain reaction (nested PCR) and loop mediated isothermal amplification (LAMP). Acta Trop. 2016;158:43-51. https:// doi.org/10.1016/j.actatropica.2016.02.005

11. Lippuner C, Ramakrishnan C, Basso WU, Schmid MW, Okoniewski M, Smith NC, et al. RNA-Seq analysis during the life cycle of Cryptosporidium parvum reveals significant differential gene expression between proliferating stages in the intestine and infectious sporozoites. Int J Parasitol.
2018;48(6):413-22. https://doi.org/10.1016/j. ijpara.2017.10.007

12. Carey CM, Lee H, Trevors JT. Biology, persistence and detection of Cryptosporidium parvum and Cryptosporidium hominis oocyst. Water Res. 2004;38(4):818-62. https://doi. org/10.1016/j.watres.2003.10.012

13. Borowski H, Thompson RCA, Armstrong T, Clode PL. Morphological characterization of Cryptosporidium parvum life-cycle stages in an in vitro model system. Parasitology. 2010;137(1):13-26. https://doi.org/10.1017/ s0031182009990837

14. Pulido-Medellín MO, Andrade-Becerra RJ, Rodríguez-Vivas RI, Garcia-Corredor DJ. Prevalencia y posibles factores de riesgo en la excreción de ooquistes de Cryptosporidium spp en bovinos de Boyacá, Colombia. Revista Mexicana de Ciencias Pecuarias. 2015;5(3):357-64

15. Eze UU, Ezeh IO, Nzeakor TA, Attama SC, Ezenduka EV, Onah DN. Prevalence and risk factors associated with Cryptosporidium spp. infection in local breed of dogs in Enugu State, Nigeria. Vet World. 2019;12(5):729-34. https:// dx.doi.org/10.14202/vetworld.2019.729-734 
16. Hastutiek P, Yuniarti WM, Djaeri M, Lastuti NDR, Suprihati E, Suwanti LT. Prevalence and diversity of gastrointestinal protozoa in Madura cattle at Bangkalan Regency, East Java, Indonesia. Vet World. 2019;12(2):198-204. https:// dx.doi.org/10.14202/vetworld.2019.198-204

17. Kotloff KL, Nataro JP, Blackwelder WC, Nasrin D, Farag TH, Panchalingam S, et al. Burden and aetiology of diarrhoeal disease in infants and young children in developing countries (the Global Enteric Multicenter Study, GEMS): a prospective, case-control study. Lancet. 2013;382(9888):209-22. https://doi. org/10.1016/S0140-6736(13)60844-2

18. Rossle NF, LatifB. Cryptosporidiosis as threatening health problem: A review. Asian Pacific Journal of Tropical Biomedicine. 2013;3(11):916-24. https:// dx.doi.org/10.1016/S2221-1691(13)60179-3

19. Vanathy K, Parija SC, Mandal J, Hamide A, Krishnamurthy S. Cryptosporidiosis: A mini review. Trop Parasitol. 2017;7(2):72-80. https://doi.org/10.4103/tp.TP_25_17

20. Attili SVS, Gulati AK, Singh VP, Varma DV, Rai $M$, Sundar S. Diarrhea, CD4 counts and enteric infections in a hospital - based cohort of HIV-infected patients around Varanasi, India. BMC Infect Dis. 2006;6(39):1-8. https://doi. org/10.1186/1471-2334-6-39
21. Dwivedi KK, Prasad G, Saini S, Mahajan S, Lal S, Baveja UK. Enteric opportunistic parasites among HIV infected individuals: associated risk factors and immune status. Jpn J Infect Dis. 2007;60(2-3):76-81

22. Muthusamy D, Rao SS, Ramani S, Monica B, Banerjee I, Abraham OC, et al. Multilocus genotyping of Cryptosporidium sp. isolates from human immunodeficiency virus-infected individuals in South India. J Clin Microbiol. 2006;44(2):632-4. https://doi.org/10.1128/ JCM.44.2.632-634.2006

23. Chavez MA, White AC. Novel treatment strategies and drugs in development for cryptosporidiosis. Expert Rev Anti Infect Ther. 2018;16(8):655-61. https://doi.org/10.1080/1 4787210.2018 .1500457

24. Tzipori S, Widmer G. A hundred-year retrospective on cryptosporidiosis. Trends Parasitol. 2008;24(4):184-9. https://dx.doi. org/10.1016/j.pt.2008.01.002

25. Darlan DM, Rozi MF, Andriyani $Y$, Yulfi $H$, Saragih RH, Nerdy N. Cryptosporidium Sp. Findings and Its Symptomatology among Immunocompromised Patients. Open Access Maced J Med Sci. 2019;7(10):1567-71. https:// doi.org/10.3889/oamjms.2019.329 
26. Ehigiator HN, Mcnair N, Mead JR. IL-12 Knockout C57BL/6 Mice are Protected from Re-infection with Cryptosporidium parvum after Challenge. J Eukaryot Microbiol. 2003;50(s1):539-41. https://doi. org/10.1111/j.1550-7408.2003.tb00622.x

27. Yu JR, Park WY. The effect of gamma-irradiation on the viability of Cryptosporidium parvum. J Parasitol. 2003;89(3):639-42. https://doi.org/10.1645/00223395(2003)089[0639:TEOIOT]2.0.CO;2

28. Jenkins M, Higgins J, Kniel K, Trout J, Fayer R. Protection of calves against cryptosporiosis by oral inoculation with gamma-irradiated Cryptosporidium parvum oocysts. J Parasitol. 2004;90(5):1178-80. https://doi.org/10.1645/ GE-3333RN

29. Wang C, Luo J, Amer S, Guo Y, Hu Y, Lu Y, et al. Multivalent DNA vaccine induces protective immune responses and enhanced resistance against Cryptosporidium parvum infection. Vaccine. 2010;29(2):323-8. https://doi. org/10.1016/j.vaccine.2010.10.034

30. Zheng J, Ren W, Pan Q, Wang Q, elhag IAE, Li $J$, et al. A recombinant DNA vaccine encoding C. andersoni oocyst wall protein induces immunity against experimental C. parvum infection. Vet Parasitol. 2011;179(1-3):7-13. https://doi.org/10.1016/j.vetpar.2011.02.016
31. Yu Q, Li J, Zhang X, Gong P, Zhang G, Li S, et al. Induction of immune responses in mice by a DNA vaccine encoding Cryptosporidium parvum Cp12 and Cp21 and its effect against homologous oocyst challenge. Vet Parasitol. 2010;172(1-2):1-7. https://doi.org/10.1016/j. vetpar.2010.04.036

32. Liu K, Zai D, Zhang D, Wei Q, Han G, Gao $H$, et al. Divalent Cp15-23 vaccine enhances immune responses and protection against Cryptosporidium parvum infection. Parasite Immunology. 2010;32(5):335-44. https://doi. org/10.1111/j.1365-3024.2009.01191.x

33. Sagodira S, lochmann S, Mevelec MN, DimierPoisson I, Bout D. Nasal immunization of mice with Cryptosporidium parvum DNA induces systemic and intestinal immune responses. Parasitelmmunol.1999;21(10):507-16.https:// doi.org/10.1046/j.1365-3024.1999.00247.x

34. He H, Zhao B, Liu L, Zhou K, Qin X, Zhang $Q$, et al. The Humoral and Cellular Immune Responses in Mice Induced by DNA Vaccine Expressing the Sporozoite Surface Protein of Cryptosporidium parvum. DNA Cell Biol. 2004;23(5):335-9. https://doi. org/10.1089/104454904323090967

35. Ehigiator HN, Romagnoli $P$, Priest JW, Secor WE, Mead JR. Induction of murine immune 
responses by DNA encoding a $23-\mathrm{kDa}$ antigen of Cryptosporidium parvum. Parasitol Res. 2007;101(4):943-50. https://doi.org/10.1007/ s00436-007-0565-0

36. Benítez A, Priest JW, Ehigiator $H N$, McNair N, Mead JR. Evaluation of DNA encoding acidic ribosomal protein P2 of Cryptosporidium parvum as a potential vaccine candidate for cryptosporidiosis. Vaccine. 2011;29(49):9239-45. https://doi. org/10.1016/j.vaccine.2011.09.094

37. Khan KH. DNA vaccines: roles against diseases. GERMS. 2013;3(1):26-35. https://dx.doi. org/10.11599/germs.2013.1034

38. Ludington JG, Ward HD. Systemic and Mucosal Immune Responses to CryptosporidiumVaccine Development. Curr Trop Med Rep. 2015;2(3):171-80. https://dx.doi.org/10.1007/ s40475-015-0054-y

39. Carleton HA. Pathogenic bacteria as vaccine vectors: teaching old bugs new tricks. Yale J Biol Med. 2010;83(4):217-22.

40. Benítez AJ, McNair N, Mead JR. Oral immunization with attenuated Salmonella enterica serovar Typhimurium encoding Cryptosporidium parvum $\mathrm{Cp} 23$ and $\mathrm{Cp} 40$ antigens induces a specific immune response in mice. Clin Vaccine Immunol. 2009;16(9):12728. https://doi.org/10.1128/CVI.00089-09

41. Manque PA, Tenjo F, Woehlbier U, Lara $A M$, Serrano $M G, X u P$, et al. Identification and Immunological Characterization of Three Potential Vaccinogens against Cryptosporidium Species. Clin Vaccine Immunol. 2011;18(11):1796-802. https://doi. org/10.1128/CVI.05197-11

42. Shirafuji $H$, Xuan X, Kimata I, Takashima $Y$, Fukumoto $S$, Otsuka $H$, et al. Expression of P23 of Cryptosporidium parvum in Toxoplasma gondii and Evaluation of its Protective Effects. Journal of Parasitology. 2005;91(2):476-9. https://doi.org/10.1645/GE-364R1

43. Stevens TL, Bossie A, Sanders VM, FernandezBotran R, Coffman RL, Mosmann TR, et al. Regulation of antibody isotype secretion by subsets of antigen-specific helper $\mathrm{T}$ cells. Nature. 1988;334(6179):255-8. https://doi. org/10.1038/334255a0

44. Lemieux $M$, Sonzogni-Desautels $K$, Ndao M. Lessons Learned from Protective Immune Responses to Optimize Vaccines against Cryptosporidiosis. Pathogens. 2017;7(1):2. https://dx.doi.org/10.3390/ pathogens7010002 
45. Geriletu, Xu R, Jia H, Terkawi MA, Xuan $X$, Zhang $H$. Immunogenicity of Orally Administrated Recombinant Lactobacillus casei Zhang Expressing Cryptosporidium parvum Surface Adhesion Protein P23 in Mice. Curr Microbiol. 2011;62(5):1573-80. https:// doi.org/10.1007/s00284-011-9894-4

46. Kwok LY, Wang L, Zhang J, Guo Z, Zhang H. A pilot study on the effect of Lactobacillus casei Zhang on intestinal microbiota parameters in Chinese subjects of different age. Benef Microbes. 2014;5(3):295-304. https://doi. org/10.3920/BM2013.0047

47. Sobati $H$, Jasor-Gharebagh $H$, Honari $H$. Expression and Purification of gp40/15 Antigen of Cryptosporidium parvum Parasite in Escherichia coli: an Innovative Approach in Vaccine Production. Iranian Red Crescent Medical Journal. 2017;19(4). http://dx.doi. org/10.5812/ircmj.43040

48. Ebrahimzadeh E, Shayan P, Mokhber Dezfouli M, Rahbari S. Recombinant Cryptosporidium parvum p23 as Candidate Vaccine for Cryptosporidiosis. Iranian Journal of Parasitology. 2009;4:1-7.

49. Zhang S, Wang Y, Wu H, Li N, Jiang J, Guo $Y$, et al. Characterization of a Species-Specific Insulinase-Like Protease in Cryptosporidium parvum. Front Microbiol 2019;10. https:// dx.doi.org/10.3389/fmicb.2019.00354

50. Avendaño $C$, Jenkins $M$, Méndez-Callejas G, Oviedo J, Guzmán F, Patarroyo MA, et al. Cryptosporidium spp. CP15 and CSL proteinderived synthetic peptides' immunogenicity and in vitro seroneutralisation capability. Vaccine. 2018;36(45):6703-10. https://doi. org/10.1016/j.vaccine.2018.09.044 